\title{
THE DIFFERENCIES IN ADULT AND PEDIATRIC MYELODYSPLASTIC SYNDROME: A REVIEW
}

\author{
Vasekova P ${ }^{1}$, Plank L. ${ }^{1,2}$ \\ ${ }^{1}$ Comenius University in Bratislava, Jessenius Faculty of Medicine and University Hospital in Martin, \\ Department of Pathological Anatomy, Slovakia, ${ }^{2}$ Martin s Biopsy Center, Ltd in Martin, Slovakia
}

\begin{abstract}
Myelodysplastic syndrome (MDS) represent very heterogenous group of clonal stem cell bone marrow disorders with ineffective haematopoesis leading to cytopenias in peripheral blood and increased risk of blastic transformation and evolution of acute myeloid leukemia. MDS is a disease of older age mostly, in children it seems to be very rare. There are several significant morphological, cytogenetic and prognostic differencies of the disease in adults and in children. Adult MDS patients most commonly manifest with symptoms of anemia, bleeding and infection are uncommon. In childhood, MDS manifests predominantly by neutropenia and thrombocytopenia. In addition, some pediatric MDS patients present also with constitutional disease's signs and symptoms. Early and correct diagnosis in both age groups is essential for the choice of appropriate therapy and also for next life of patients. However, the diagnosis of MDS is challenging, complex and requiring close correlation of clinical symptoms, laboratory parameters and standardized examination of BM biopsies. The authors present an overview focused on biology of MDS in adults and children, on the differences in the incidence, clinical presentation and treatment. They summarize the possibilities and limits of histopathological diagnosis and differential diagnosis of the disease in different age groups. A major problem in the morphological diagnosis of MDS remains the determination, whether the myelodysplasia is due to clonal disorder. It might result also from some other factors, as significant dysplasia can also occur in reactive conditions, and vice versa, only discrete dysplasia is sometimes observed in MDS patients. Although histomorphological and immunohistochemical analysis of BM biopsy is invasive and time-consuming examination, it has its value in the diagnosis, differential diagnosis and evaluation of therapeutic effect.
\end{abstract}

Key words: Myelodysplastic syndrome, types of myelodysplastic syndrome, risk stratification, adults, children

\section{INTRODUCTION}

Myelodysplastic syndromes (MDS) represent group of clonal stem cell bone marrow (BM) disorders with ineffective haematopoesis leading to cytopenias in peripheral blood (PB) and increased risk of blastic transformation and evolution of acute myeloid leukemia (AML) $(1,2)$. They are very heterogenous myeloid malignancies, mainly of older age, with slightly predominant incidence in men (excluding type with isolated deletion of 5q) (3). At diagnosis, median age is 65-70 years, the incidence increases with age ( less than $1 / 100000$ before 50 years, more than 20/100 000 after 70$)(4,3)$. MDS is rare haematooncological disease in children, typically occuring in age of 6-8 years. According to WHO classification (2008), pediatric MDS accounts for less than 5\% of all haematological malignancies in childhood. The estimated incidence is reported 0,5-4 / 1 million yearly (5), although this statement may be distorted due to difficulties with diagnosis of pediatric MDS (6). There are several significant morphological, cytogenetic and prognostic differencies of disease in adults and in children. $(6,7)$. Tab 1

\section{CLASSIFICATIONS:}

The current WHO classification (2008) distinguishes these MDS subtypes of adults: Refractory cytopenia with unilinear dysplasia (RCUD) manifests by refractory cytopenia

Address for correspondence:

Petra Vašeková, MD., Department of Pathological Anatomy, Comenius University in Bratislava, Jessenius Faculty of Medicine and University Hospital in Martin, Kollárova 2, 03601 Martin, Slovakia; Phone: (+421)-434133002; Fax 043/420 33 70; e-mail: p.vasekova@yahoo.com 
Table 1. Comparison of the most common features of adult and pediatric MDS (according to Glaubach T., Robinson L. J., Corey S. J., 2014 and Chatterjee T. Choudhry V. P., 2013)

\begin{tabular}{|c|c|c|}
\hline & Adult MDS & Pediatric MDS \\
\hline Incidence & $3-5 / 10^{5},>20 / 10^{5} u>70 r$ & $1.8-4 / 10^{6}$ \\
\hline $\begin{array}{l}\text { Median age } \\
\text { (years) }\end{array}$ & 70 & 7 \\
\hline Presentation & $\begin{array}{l}\text { isolated anemia with / without neu- } \\
\text { tropenia and / or trombocytopenia }\end{array}$ & $\uparrow$ bicytopenia - RT > RN and / or anemia \\
\hline Etiology & $\uparrow$ primary (de novo) & $\uparrow$ secondary and t-MDS \\
\hline $\begin{array}{l}\text { Morphological } \\
\text { subtype }\end{array}$ & $\begin{array}{l}\uparrow \text { RARS a } 5 q \text { - syndrome, without } \\
\text { RAEB-T, subclassification in RAEB1 } \\
\text { and } 2 \text { with prognostic significance }\end{array}$ & $\begin{array}{l}\uparrow \text { RCC, RARS and } 5 q-\text { syndrome are rare, } \\
\text { RAEB-T is used, without data about signifi- } \\
\text { cance of subclassification in RAEB } 1 \text { and } 2\end{array}$ \\
\hline BM cellularity & $\uparrow$ or normal, rare & variable, hypocellular \\
\hline Cytogenetics & $5 / 5 q->7 / 7 q^{-}, 3 q$ a $20 q$ anomalies & $-7 / 7 q>+8$ \\
\hline Genetics & $\begin{array}{l}\text { mutations in DNMT3A, ASXL1, } \\
\text { TET2, SF3B1, U2AF5, methylating } \\
\text { changes }\end{array}$ & $\begin{array}{l}\text { mutations in FANC, SBDS, DKC, TERT, } \\
\text { TERC, ELANE, HAX1, WAS, GATA2 }\end{array}$ \\
\hline $\begin{array}{l}\text { Physical } \\
\text { findings }\end{array}$ & nono & $\begin{array}{l}\text { skeletal, cutaneous, genitourinary, cardio- } \\
\text { vascular and gastrointestinal anomalies } \\
\text { (related to IBMFS) }\end{array}$ \\
\hline $\begin{array}{l}\text { Therapeutic } \\
\text { options }\end{array}$ & transfusions, LND, HA, HSCT & $\begin{array}{l}\text { monitoring, transfusions, HSCT } \\
\text { ? LND, ? HA }\end{array}$ \\
\hline
\end{tabular}

\section{Legend:}

RT - refractory thrombocytopenia

RARS - refractory anemia with ring sideroblasts

$\mathrm{RN}$ - refractory neutropenia

RAEB / RAEB-T - refractory anemia with excess blasts / in transformation

\section{-7 - monosomy 7}

LND - lenalidomid

HSCT - haemopoetic stem cell transplantation

IBMFS - inherited bone marrow failure syndromes
+8 - trisomy 8

HA - hypomethylating agents

IST - immunosuppressive therapy

? - unknown efficacy

and dysplasia limited to one cellular lineage and includes the most common refractory anemia (RA) and less frequent refractory neutropenia (RN) and thrombocytopenia (RT). It accounts for around 10-20\% of diagnosed MDS cases (5). Refractory anemia with ring sideroblasts (RARS) is characterised by unexplained anemia, dysplastic changes affecting erythroid lineage and presence of more than $15 \%$ of ring sideroblasts. It represents about $3-11 \%$ of all cases (5).

Refractory cytopenia with multilinear dysplasia (RCMD) represents MDS with one or more cytopenia and dysplasias in two or more myeloid lineages. This subgroup accounts for around 30\% of all MDS (5).

Refractory anemia with excess blasts (RAEB) is characterised by presence of 5-19\% of myeloblasts in BM and 2-19\% of myeloblasts in PB. According to the blasts count, two clinically different subtypes of RAEB has to be recognized due to different patient's survival and 
incidence of leukemic evolution: RAEB1 (5-9 \% blasts in BM and 2-4\% blasts in PB) and RAEB2 (10-19\% blasts in BM and 5-19\% in PB) $(5,8)$.

MDS with isolated deletion of $5 q$ (s.c. $5 q$ - syndrome) is characterised by macrocytic anemia with or without other cytopenia and / or thrombocytosis with the sole cytogenetic abnormality - deletion of 5q, it typically afects elderly women (5).

MDS unclassifiable represents those cases which can not be classified to any of the preceding groups. These patients should be monitored regularly in order to recognize the evolution of the disease in a more specific type (5).

According to the WHO classification (2008), pediatric MDS are classified as follows:

Refractory cytopenia of childhood (RCC) is the most common type with variable clinical course (9), accounting for approximately half of all MDS cases (6). It is characterised by persistent cytopenia with less than 5\% BM blasts, less than $2 \%$ of PB blasts and dysplastic changes in two or three lineages or exceeding more than $10 \%$ of cells in one lineage (9). In contrast to adult MDS, the WHO classification (2008) does not specify the importance of the number of lineages involved in the dysplasia (which has prognostic significance in adults with MDS). It is recommended, that children who meet the criteria for RCMD, should be considered as RCC, until the prognostic significance of a multilineage presentation is further clarified in children (10).

Advanced pediatric MDS - RAEB (PB blasts 2-19\% and/or BM blasts 5-19\%), RAEB in transformation (RAEB-T, PB and/or BM blasts 20-29\%).

Pediatric RAEB exhibits similar morphological and immunohistochemical features as in adults, however, children have relative stabile blood count for weeks to months $(11,12)$. The progression to secondary AML is variable, from slow period of several months to sudden transformation (7). It occurs in about 30\% of cases of pediatric MDS, usually up to 2 years from diagnosis (13). In contrast to adult MDS, significance of subclassification into RAEB1 and RAEB 2 is not clear $(14,15)$.

\section{PATHOGENESIS}

According to pathogenesis, there are two types of MDS: primary or de novo and secondary. Despite extensive studies, particulary in pediatric cases, the causes of origin and progression of primary MDS are not yet fully understood. Pathogenesis of adult and pediatric MDS is multifactorial (16), as the ineffective haematopoesis may be caused by heterogenous defects of BM stem cells. It is postulated, that genetic defects in the pluripotent progenitor cells lead to genetic instability with consequent numerous molecular and cellular abnormalities (7). Also excessive BM apoptosis plays role in pathogenesis (6). The partial therapeutic response on immunosuppression and autoimmune diseases symptoms suggest participation of deregulation of the immune system. In general, a lot of mechanisms take a part in pathogenesis with deregulation of cellular proliferation, differentiation, maturation and survival $(17,8)$. Especially genetic instability, epigenetic events, abnormal signal transduction, immune deregulation and finally BM microenvironment seem to have the most important role (18). Secondary MDS occuring after previous chemotherapy and / or radiotherapy is called "therapy-related“. Its incidence is growing due to longer survival of patients. Secondary MDS in childhood can arise in young patients with inherited BM failure disorders (IBMFS) (12). IBMFS represent a small and rare, but important group of diseases with risk of MDS evolution (19) characterised by failure of haematopoetic stem cell to produce blood cells with PB cytopenias (20). It is estimated that about $30 \%$ children with MDS share also constitutive disorder (7) Tab 2.

Also some others pediatric genetically determined diseases are associated with higher risk of MDS developement, such as MonoMac syndrome, Down`s syndrome, neurofibromatosis, Bloom syndrome or Li-Fraumeni syndrome. In some of these cases, MDS represents the first presenting sing of the disease (15). The risk of clonal evolution and developement of secondary MDS in aplastic anemia in children is high, as it occurs in about 25-40\% of cases (21). 


\section{PROGNOSIS}

Searching for prognostic variables for reliable stratifications of MDS patients, predictions of mortality and blastic transformation has started approx. in 1980 (22). Several prognostic scoring systems have been proposed, among them the most important and widely used are IPSS and IPSS-R. Clinical course of the disease is variable, but without therapy and often also despite treatement it is fatal (23). The median survival of patients with de novo MDS is about 2 years. Almost 30\% of MDS patients die due to secondary AML, $40 \%$ due to $\mathrm{BM}$ failure complications such as infections, bleeding or iron overload and last $30 \%$ of patient due to cardiovascular complications and non-haematological malignancies. The median survival in therapy - related MDS is only about 3-8 months. The higher age of patients and associated comorbidities contribute to adverse prognosis $(24,4)$. There were several attempts to create a prognostic score of pediatric MDS, but none of them has been so far significantly applied in clinical practice, also IPSS shows only a limited value $(14,6)$.

Adult and also pediatric MDS can be divided into chronic low risk disease with slow progression (25) and more aggressive high risk with rapid leukemic evolution and short survival $(26,27)$.

Table 2. IBMFS, mutated genes and estimated prevalence of myeloid neoplasms (according to Niemeyer Ch. M., Kratz Ch. P., 2008).

\begin{tabular}{|l|l|l|}
\hline \multicolumn{1}{|c|}{ Disease } & \multicolumn{1}{|c|}{ Mutated genes } & \multicolumn{1}{c|}{$\begin{array}{c}\text { Frequency of myeloid } \\
\text { malignancy }\end{array}$} \\
\hline Fanconi anemia & 1 from 13 FANC genes & $30-40 \%$ \\
\hline Diamond-Blackfan anemia & RPS19, RPS24, RPS17 & $5 \%$ \\
\hline Kostman`s disease & HAX1, ELA2, GFI1, WAS & $30 \%$ \\
\hline Schwachman-Diamond syndrome & SBDS & $30-40 \%$ \\
\hline Dyskeratosis congenita & DKC1, TERC, TERT, NOLA3 & $5 \%$ \\
\hline FTD/AML* & RUNX1 & $?$ \\
\hline
\end{tabular}

Legend: *familial thrombocytopenia with propensity to MDS and AML

\section{CLINICAL FEATURES}

Most common manifestation of MDS of adult patients includes signs representing consequences of anemia (e.g. such as weakness, fatigue, palpitations etc.), while bleeding and infections are rare. Arthralgia or intermittent fever may be present in some patients. However, in about $50 \%$ of cases the early stages of the disease are asymptomatic $(28,3)$. MDS is suspected in cases with persistent cytopenia lasting more than 6 months, when other possible causes of cytopenia are excluded (29). In childhood, MDS manifests predominantly by neutropenia and thrombocytopenia (6), in a portion of children also constitutional disease's signs and symptoms may be present.

\section{DIAGNOSIS}

Early and correct diagnosis is essential for the choice of appropriate therapy and also for next life of patients. However, the diagnosis of MDS is challenging, complex and requiring close correlation of clinical symptoms, laboratory parameters and standardized examination of BM biopsies. It often represents a diagnosis „per exclusionem“ $(28,24)$. The current 
diagnostic algorithm (as well as classification and prognostification of patients) is based on clinical parameters, the analysis of peripheral blood, BM aspirate and cytogenetic (4), but careful histological and immunohistochemical examination of BM biopsy is often necessary for the final diagnosis and precise classification of the patients (30).

For a correct interpretation of morphological and immunohistochemical findings by pathologist examining the BM biopsy, it is essential to have access to clinical data of the disease, laboratory findings, complete blood counts, results of flow cytometry and of cytogenetics (30).

Typical histomorphological MDS findings in BM biopsies of adults are represented by a distortion of BM microarchitecture, cytological changes and of topographical conditions, all they include (31):

1. Apparently unequal distribution of fat cells and thus different cellularity in various intertrabecular areas. In most cases, the BM is hypercellular, although there are also cases with a relatively normocellular BM. About $10 \%$ of cases show so-called hypoplastic MDS with reduced BM cellularity (5). However, it is important to assess the BM cellularity in comparison to the patient s age,

2. significant differences in the size of hematopoietic islets,

3 . increase of BM stromal reticulin fibers of varying grades,

4. a significant increase in the proportion of ring sideroblasts,

The ring sideroblasts are the red cells precursors with the abnormal accumulation of iron in the mitochondrias, which contain at least 5 siderotic granules encircling one third or more of the nucleus (24). They are considered as part of dysplastic changes in MDS, but can be detected also in other disorders and reactive changes as well, thus again they are not specific (24).

5. left shifted maturation of granulopoiesis, creating the „Abnormal Localization of Immature Precursors“ (ALIP) phenomenon, characterized by clusters of less mature forms of granulocyte development in the interstitium, including bone's trabecula and vascular structures.

6. varying degrees of erythropoiesis hyperplasia changing the physiological topographic distribution of its precursors - a typical feature, although somewhat unspecific, is dislocation of red cell precursors to paratrabecular areas. Typical but not exclusivelly specific features of MDS include anisocytosis and macrocytic and megaloblastic differentiation of erythroid precursors. Dysplastic changes may be nuclear (budding and/or bridging, karyorrhexis, multinuclearity and megaloblastoid changes) or cytoplasmic (presence of ring sideroblasts, vacuolization and periodic acid-Schiff positivity). The increased mitotic activity associated with maturation arrest is characteristic too $(32,28,5)$. The assessment of dysplastic changes in both white and red cell lineages should be preferantially done in the BM aspirate as they are hardly detectable in BM biopsies,

7. abnormal cytomorphology and localization of megakaryocytes (mgk)

Number of mgk in BM in the MDS can be normal, increased or even reduced, but significant hypoplasia is rare (31). A typical feature of dysplastic mgk is a tendency to form free and tight clusters, in rare cases even large cohesive infiltrates (31). In contrast dysplasias of white and red cell precursors, the BM biopsy is preferred for evaluations of cytologic atypias im mgk series (33). They are represented by changes of nuclear lobulizations, e.g. hypo, or non-lobulizated nuclei with usually dense chromatin, they may also occur as apoptotic forms $(24,32)$. Another morphological change is the multinuclearity of mgk $(28,32)$. A strong indicator of the MDS is recognition of s.c. micromegakaryocytes (micromgk) (34), but they might be unrecognized in routine histomorphologic slides. To avoid such a mistake it is recommended, to apply immunohistochemical staining identifying typical mgk CD61 and CD41 antigens (32),

8. increased vascularization of $\mathrm{BM}$, and

9. increased expression of CD34 antigen in the immature myeloid precursors.

Generally, a correct BM biopsy diagnosis of a "true“ MDS including its differential distinction from reactive conditions, necessarily requires the application of immunohisto- 
chemical methods. For the biopsy diagnosis of MDS it is recommended to apply constantly a standardized examination of expression of following antigens: CD34 (myeloblasts detection), myeloperoxidase (precursors of granulopoiesis), CD61 or CD41 (mgk and micromgk), glycophorin A (precursors of erythropoiesis), CD20 (B cells), CD3 (T cells) and of CD68 (monocytes and macrophages).

In about $75 \%$ of children with RCC the BM cellularity is decreased with peculiar patchy distribution of haematopoesis in an otherwise fatty marrow (so-called "patchy pattern") (11, 35). In these cases the essential marker of histopathological RCC diagnosis in BM biopsy is the presence of at least one island with at least 20 precursors with affected maturation and increased mitotic index. Granulopoiesis is reduced, with a possible shift to the left in maturation, but without increased proportion of myeloblasts. Elements of mgk series can be reduced or completely absent, while the finding of micromgk is pathognomonic also for MDS in children $(11,35)$. In some cases, however, morphology is different and corresponds with RCMD of adult age (36), what is sometimes described as „diffuse type“ of RCC (37).

\section{DIFFERENTIAL DIAGNOSIS}

A major problem in the morphological diagnosis of MDS is the determination, whether the presence of myelodysplasia is due to clonal disorder or results from some other factors (5). It is accepted that finding of manifesting dysplasia in more than $10 \%$ of cell of at least one cell lineage qualifies the MDS diagnosis. However, significant dysplasia may occur also in non-MDS cases and even in healthy persons too. Therefore some studies suggest that the assessment criteria might be tightened (32). Dysplastic changes alone are therefore not unique to MDS, as we know the number of diseases or factors that may cause secondary dysplasia and in adulthood. In particular, they might be summarized as follows:

1) megaloblastic anemia (MA) due to folate or vitamin B12 deficiency is one of the most common causes of secondary dysplastic changes in BM. These changes primarily affect the red lineage with megaloblastic differentiation and changes in topography of varying degrees (38). The total number of white series precursors can be easily reduced resulting in decreased physiological ratio of white and red series. Severe anemia may be associated with mild thrombocytopenia and the BM biopsy may show bizarre forms of mgk (39). Differentiation of MDS and MA based on the morphological (and immunohistochemical) BM changes is virtually impossible. Thus, clinical informations about a) levels of folic acid and vitamin $\mathrm{B} 12$, b) disorders of the stomach affecting the parietal cells (producing intrinsic factor needed for the absorption of vitamin B12 from food $(39,40)$, c) excessive abuse of alcohol (39) and finally, d) all chronic disease (infection, inflammation and malignancies) which lead to anemia of chronic disease (8) are very important and helpful.

2) chronic renal diseases can cause secondary dysplastic changes due to decreased production of erythropoietin, the primary regulator of erythropoiesis.

3) drugs can also cause BM changes that mimic MDS and induce cytopenias in PB. The most common are co-trimoxazole, mycophenolate mofetil, and other immunosuppressive agents, corticosteroids, most chemotherapeutic agents, growth factors and many other (8).

4) other - hypothyroidism, hepatopathy, haematopoietic and non-haematopoietic malignancies, autoimmune diseases, idiopathic inflammatory bowel disease, intoxication with heavy metals, virus diseases, etc. (8)

As in adults, the MDS diagnosis in children is usually morphological and therefore subjective (15). The diagnosis of pediatric MDS is challenging, especially in children with low blasts count without clonal marker, or in cases with discrete dysplasia only (14). In contrast, a significant dysplasia can also occur in reactive conditions of childhood. In pediatric disease, it is important to distinguish in particular:

1) MDS with low blasts count from aplastic anemia and other nonclonal diseases:

a) aplastic anemia - defined by aplasia of all three hematopoietic cell lineages.

Similar to most hypocellular RCC there is a marked decrease in BM cellularity below $30 \%$ for age with reduction of hematopoietic cells and substituting fatty marrow, but erythroid 
islands and micromgk are not present in BM biopsy (Tab 3. - 11, 41, 35). BM architecture is essentially preserved with persisting non-hematopoietic cells, sometimes with the impression of a chronic inflammatory infiltrate $(21,35)$. However, if the BM cellularity is significantly reduced, to assess the status of haemopoetic cells can be extremely difficult (15). Overexpression of p53 in cases of MDS detected by immunohistochemistry appears to be useful (14). For the diferential diagnosis of RCC and aplastic anemia it might be essential to repeat the BM biopsy (37) to receive and consider representative marrow spaces. (12).

b) IBMFS with pancytopenia - shows in BM biopsies similar morphology to MDS and essentially indistinguishable changes from RCC. It is therefore necessary to exclude these syndromes mainly clinically using an extensive physical examination, assessment of family history and relevant laboratory and molecular measurements $(11,35)$. Histological examination can detect the progression of the diseases to secondary MDS, especially in the cases of increasing the blasts count or rising BM cellularity with progressive pancytopenia in the PB $(11,14)$.

c) secondary myelodysplasia due to viral infections (EBV, CMV, parvovirus), rheumatic diseases (juvenile idiopathic arthritis) or nutritional deficiencies (15).

Table 3. Morphological differencies between refractory cytopenia of childhood and aplastic anemia (according to Baumann I., Führer M., Behrendt S., et al., 2012 and Chatterjee T. Choudhry V. P., 2013)

\begin{tabular}{|l|l|l|}
\hline & Refractory cytopenia of childhood & aplastic anemia \\
\hline Erythropoesis & $\begin{array}{l}\text { Patchy distribution } \\
\text { Left shifted maturation } \\
\text { Increase mitoses }\end{array}$ & $\begin{array}{l}\text { Lacking foci or a single small focus } \\
\text { with <10 cells with maturation }\end{array}$ \\
\hline Granulopoiesis & $\begin{array}{l}\text { Marked decrease } \\
\text { Left shift }\end{array}$ & $\begin{array}{l}\text { Lacking or marked decrease, very few } \\
\text { small foci with maturation }\end{array}$ \\
\hline Megakaryopoiesis & $\begin{array}{l}\text { Marked decrease } \\
\text { Mysplastic changes } \\
\text { Micromegakaryocytes }\end{array}$ & $\begin{array}{l}\text { Lacking or very few, no dysplastic } \\
\text { megakaryocytes }\end{array}$ \\
\hline Lymphocytes & May be increased focally or dispersed & May be increased focally or dispersed \\
\hline CD34+ cells & No increase & No increase \\
\hline
\end{tabular}

2) MDS with excess blasts from the primary, de novo AML.

There are significant differences in clinical features, cytogenetics and in response to therapy between MDS and de novo AML reflecting fundamental biological differences (7). Primary AML characterized by a specific recurrent translocations is chemosensitive, on the contrary, MDS and secondary AML are chemoresistant and defined by numerous chromosomal abnormalities (14). In contrast to adults, it is not yet well-defined boundary threshold of blast percentage to separate RAEB from AML. That was the reason to maintain in the classification of pediatric MDS the subgroup RAEB in transformation („RAEB-T“), with the proportion of blasts in BM between 20-29\%. In such borderline cases with dysplasia and in the absence of cytogenetic markers, it is recommened to repeat the BM examination two weeks later. If the blast count has increased above 30\%, or organomegaly or a significant leucocyte increase in the PB has appeared, it is most likely the condition represents a primary AML (7). If the blast count is stable, then an arbitrary period of 4 weeks is suggested before establishing a diagnosis of RAEB-T. However, most children with primary AML present from the beginning as a frank AML (14). 
In the presence of specific translocations, e.g. t $(8 ; 21)(\mathrm{q} 22 ; \mathrm{q} 22)$ and $\mathrm{t}(15 ; 17)$ (q22; q12), or inversion, for example inv16 (p13; q22), the disease should be considered as AML, irrespectively of the blast proportion in BM $(7,14)$.

\section{TREATMENT}

Differences between adult and pediatric MDS are also reflected in therapy options. There are numbers of therapeutic procedures (supportive, stimulating, immunomodulatory with lenalidomide) in adult patients with low and intermediate risk disease, which the main aim to extend the life of hematopoietic cells (42). In patients at high risk, the dominating effort is to delay leukemic evolution by hypomethylating agents, intensive chemotherapy and transplantation of haematopoietic stem cells (33). Compared to the adult population, a major therapeutic goal in childhood is to cure pediatric patients (15) and the only potentially curative option remains transplantation of haematopoietic stem cells. In some pediatric patients, immunosuppressive therapy may be effective (10). Hypomethylating agents have the potential to modify the course of the disease and have become a standard in the treatment of disease in the adult patient population, but the effects in children still remains unclear $(43,15)$.

\section{CONCLUSIONS}

MDS both in adults and in children is a severe disease, regardless of subtype. Diagnosis, classification and selection of optimal treatments are currently based on a comprehensive evaluation of the patient, including assessment of morphological, immunophenotypic and cytogenetic findings. Although histomorphological and immunohistochemical analysis of BM biopsy is invasive and time-consuming examination, it has its value in the diagnosis, differential diagnosis and evaluation of therapeutic effect. However, clinicopathological mutual cooperation is necessary for correct interpretation of observed changes.

\section{REFERENCES}

1. THEPOT S., BOEHRER S., SEKERES V. et al. (2014). A phase I/II trial of Erlotinib in higher risk myelodysplastic syndromes and acute myeloid leukemia after azacitidine failure. Leuk Res 38: 1430-1434

2. RANKIN E. B., NARLA A., PARK J. K. et al. (2015). Biology of the bone marrow microenvironment and myelodysplastic syndromes. Mol Genet Metab 116: 24-28

3. A DES L., ITZYKSON R., FENAUX P. (2014). Myelodysplastic syndrome. Lancet 383:2239-2252

4. CAZZOLA M., DELLA PORTA M. G, TRAVAGLiNO E. et al. (2011). Classification and Prognostic Evaluation of Myelodysplastic Syndromes. Semin Oncol 38 (5): 627-634

5. SWERDLOW S. H., CAMPO E., HARRIS N.L. et al. (2008). World Health Organization Classification of Tumours of Hematopoetic and Lymphoid Tissues. 4th Edition. Lyon: IARC Press 2008: 86-103.

6. NIEMEYER CH. M., KRATZ CH. P. (2008). Paediatric myelodysplastic syndromes and juvenile myelomonocytic leukaemia: molecular classification and treatment options. Br J Haematol 140: 610-624

7. HASLE H., NIEMEYER CH. M. (2011). Advances in the prognostication and management of advanced MDS in children. Br J Haematol 154: 185-195

8. ORAZI A., CZADER B. M. (2009). Myelodysplastic Syndromes. Am J Clin Pathol 132: 290-305

9. INAGAKI J., FUKANO R., KARAUCHI K. et al. (2015). Hemopoetic stem cell transplantation in children with refractory cytopenia of childhood: Single-center experience with high dose cytarabine containing myeloablative and aplastic anemia oriented reduced - intensity conditioning regimens. Biol Blood Marrow Transplant 21 (3): 565-569

10. HASEGAWA D., CHEN X., HIRABAYASHI S. et al. (2014). Clinical characteristics and treatement outcome in 65 cases with refractory cytopenia of childhood defined according to the WHO 2008 classification. Br J Haematol 166: 758-766

11. NIEMEYER CH. M., BAUMANN I. (2011). Classification of childhood aplastic anemia and myelodysplastic syndromes. ASH Educational Book 2011 (1): 84-89

12. CHATTERJEE T., CHOUDHRY V. P. (2013). Childhood Myelodysplastic Syndrome. Indian J Pediatr 80 (9): 764-771 
13. BASQUIRA A. L., PIZZI S., CORREAS A. G. et al. (2015). Allogenic hematopoetic stem cell transplantation in pediatric MDS: A multicenter experience from Argentina. Pediatr. Blood Cancer 62: 153-157

14. HASLE H., NIEMEYER CH. M., CHESSELS J. M. et al. (2003). A pediatric approach to the WHO classification of myelodysplastic and myeloproliferative disease. Leukemia 17: 277-282

15. GLAUBACH T., ROBINSON L. J., COREY S. J. (2014). Pediatric Myelodysplastic Syndromes: They Do Exist! J Pediatr Hematol Oncol 36 (1): 1-7

16. WU J. CHENG Y., ZHANG L. (2015). Comparison of immune manifestations between refractory cytopenia of childhood and aplastic anemia in children: A single-center retrospective study. Leuk Res 39 (12): 1347-1352

17. JÄDERSTEN M., HELLSTRÖM-LINDBERG E. (2008). Myelodysplastic syndromes: biology and treatement. J Intern Med 265: 307-328

18. GREENBERG P. L. (2012). Molecular and genetic features of myelodysplastic syndrome. Int. Jnl. Lab. Hem. 34 : 215-222.

19. WEINZIERL E. P., ARBER D. A. (2013). The differential diagnosis and bone marrow evaluation of new-onset pancytopenia. AM J Clin Pathol. 139: 9-29

20. VISCONTE V., LINDSLEY R. C., BERLYNE D. et al. (2015). Aplastic Anemia \& MDS International Foundation (AA \& MDS IF): Bone Marrow Failure Disease Scientific Symposium 2014. Leuk Res 39: $110-113$

21. GORDON-SMITH E. C. (2011). Blood and Bone Marrow Pathology (Second Edition). CHURCHILL LIVINGSTONE ELSEVIER, 2011, p. 213-224, ISBN 978-0-7020-3147-2

22. KAO J. M., GREENBERG P. L. (2007). Myelodysplastic Syndromes: Impact of Recently Analyzed Variables for Modifying Current Classification Methods. Clinical Leukemia 1 (3) : 172-182

23. RADICH J. (2008). Solving the Mystery of Myelodysplasia. PLoS Med 5(2): e40

24. INVERNIZZI R., FILOCCO A. (2010). Myelodysplastic syndrome: classification and prognostic systems. Oncol Rev 4: 25-33

25. MITTELMAN M., OSTER. H. S., HOFFMAN M. at al. (2010). The lower risk MDS patient at risk of rapid progression. Leuk Res 34: 1551-1555

26. AMERI A. A., CHERRY M., GARCIA-MANNERO G. et al. (2011). Standard Therapy for Patients With Myelodysplastic Syndromes. Clin Lymphoma Myeloma Leuk 11 (4): 303-313

27. SEKERES M. A (2013). The euphoria of hypomethylating agents in MDS andAML: Is it justified? Best Pract Res Clin Haematol. 26: 275-278

28. PILO F., DI TUCCI A. A., DESSALVI P. et al. (2010). The evolving clinical scenario of myelodysplastic syndrome: The need for a complete and up to date upfront diagnostic assesment. Eur $\mathrm{J}$ Intern Med 21: 490-495

29. MALCOVATI L., HELLSTRÖM-LINDBERG E., BOWEN D. et al. (2013). Diagnosis and treatment of primary myelodysplastic syndromes in adults: recommendations from the European LeukemiaNet. Blood 122 (17) : 2943-2964

30. VALENT P., ORAZIS A., BÜSCHE G. et al. (2010). Standards and Impact of Hematopathology in Myelodysplastic Syndromes (MDS). Oncotarget 1: $483-496$

31. HORNY H.-P., SOTLAR K., VALENT. P. (2007). Diagnostic value of histology and immunohistochemistry in myelodysplastic syndromes. Leuk Res 31: 1609-1616

32. HASSERIJAN R. P. (2015). Advances in the diagnosis and classification of myelodysplastic syndromes. Diagn Histopathol 21 (5): 203-211

33. MEERS S. (2014). The myelodysplastic syndromes: the area of understanding. Eur J Haematol 94 : 379-390

34. GIAGOUNIDIS A., HAASE D. (2013). Morphology, cytogenetics and classification of MDS. Best Pract Res Clin Haematol 26 : 337-353

35. BAUMANN I., FÜHRER M., BEHRENDT S. et al. (2012). Morphological differentiation of severe aplastic anemia from hypocellular refractory cytopenia of childhood: reproducibility of histopathological diagnostic criteria. Histopathology 61: 10-17

36. HAMA A., MANABE A., HASEGAWA D. et al. (2015). Comparison of clinical features between aplastic anemia and refractory cytopenia of childhood. Haematologica 100 (s2) : p2

37. BAUMANN I. (2015). Morphologic diagnosis of RCC vs. SAA. Oral presentation- Keynote Lecture. 7th International Symposium on Myelodysplastic Syndromes, JMML and Bone Marrow Failure, Aarhus, Denmark, 1-3 October 2015

38. FOUCAR K., VISWANATHA D.S., WILSON C. S. (2008). Non neoplastistic disorders of bone marrow, First series, Fascicle 6. Washington DC: ARP Press 2008: 84-89 
39. PARKER-WILLIAMS E. (2009). Investigation and management of anaemia. Medicine 37 (3): 137-142

40. BIZZARO N., ANTICO A. (2014). Diagnosis and classification of pernicious anemia. Autoimmun Rev 13: 565-568

41. BARONE A., LUCARELLI A., ONOFRILLO D. et al. (2015). Diagnosis and management of Acquired Aplastic Anemia in Childhood. Guidelines from the Marrow Failure Study Group of the Pediatric Heamato-Oncology Italian Association (AIEOP). Blood Cells Mol Dis 55 (1): 40-47

42. GARCIA-MANERO G. (2015). Myelodysplastic syndromes: 2015 Update on diagnosis, risk-stratification, and management. Am. J. Hematol. 90 (9): 832-840

43. SMITH A. R., CHRISTIANSEN E. C., WAGNER J. E. et al. (2013). Early Hematopoetic Stem Cell Transplant Is Associated With Favorable Outcomes In Children With MDS. Pediatr. Blood Cancer 60: 705-710

Received: April,26,2016

Accepted: July,15,2016 